\title{
ALONGAMENTO DO PROCESSO ESTILÓIDE (SÍnDROME DE EAGLE): RELATO DE DOIS CASOS*
}

\author{
Antonio Carlos Domingues de Sá1, Maurício Zardo², Ademar José de Oliveira Paes Junior ${ }^{3}$, \\ Ricardo Pires de Souza ${ }^{4}$, Francisco Barros Neto ${ }^{5}$, Marcelo de Oliveira Dreweck ${ }^{6}$, Regiane \\ de Oliveira ${ }^{7}$, Murilo Postiglioni Neme ${ }^{8}$, Abrão Rapoport ${ }^{9}$
}

\begin{abstract}
Resumo 0 alongamento do processo estilóide, ou síndrome de Eagle, representa uma afecção multifatorial com características inespecíficas na análise do quadro clínico e no estudo por imagem. A utilização dos métodos de imagem, em associação aos sinais e sintomas, é de grande utilidade na confirmação diagnóstica, mostrando a extensão do complexo estilóide, os músculos e ligamentos que o compõem e as estruturas adjacentes. $O$ diagnóstico é firmado quando se tem a associação das informações clínicas com os exames de imagem. Os autores descrevem dois casos de alongamento do processo estilóide e seus aspectos de imagem, principalmente por meio da tomografia computadorizada.

Unitermos: Síndrome de Eagle; Diagnóstico; Tomografia computadorizada.
\end{abstract}

Abstract Elongated styloid process (Eagle syndrome): a case report.

Elongated styloid process or Eagle's syndrome is a multifactorial disease with unspecific characteristics on clinical and imaging examinations. The use of imaging methods in combination with the signs and symptoms are valuable in the confirmation of the diagnosis since they show the extension of the styloid complex, the muscles and ligaments, and adjacent structures. The diagnosis can be established when there is an association of clinical information and imaging findings. The authors report two cases of elongated styloid process including the imaging findings, mainly obtained using computed tomography.

Key words: Eagle's syndrome; Diagnosis; Computed tomography.

\footnotetext{
* Trabalho desenvolvido no Serviço de Cirurgia Buco-maxilofacial do Hospital Santa Casa de Misericórdia de Ponta Grossa, Ponta Grossa, PR.

1. Especialista em Cirurgia Buco-maxilo-facial pela Universidade de São Paulo - USP-Bauru, Membro do Serviço de Cirurgia Maxilo-facial do Hospital Santa Casa de Misericórdia de Ponta Grossa, Mestrando do Curso de Pós-graduação em Ciências da Saúde do Hospital Heliópolis, Professor da Pós-graduação da Escola de Aperfeiçoamento Profissional da Associação Brasileira de Odontologia Ponta Grossa.

2. Doutor em Cirurgia Buco-maxilo-facial pela Universidade do Estado de São Paulo - Unesp-Araçatuba, Membro do Serviço de Cirurgia Maxilo-facial do Hospital Santa Casa de Misericórdia de Ponta Grossa, Coordenador da Equipe de Pós-graduação em Cirurgia Buco-maxilo-facial da Escola de Aperfeiçoamento Profissional da Associação Brasileira de Odontologia Ponta Grossa, Professor Adjunto de Cirurgia da Universidade Estadual de Ponta Grossa.

3. Médico Residente em Radiologia e Diagnóstico por Imagem do Complexo Hospitalar Heliópolis, Mestrando do Curso de Pósgraduação em Ciências da Saúde do Hospital Heliópolis.

4. Doutor em Radiologia pela Faculdade de Medicina da Universidade de São Paulo, Professor do Curso de Pós-graduação em Ciências da Saúde do Hospital Heliópolis, Coordenador do Programa de Residência Médica em Radiologia e Diagnóstico por Imagem do Complexo Hospitalar Heliópolis.

5. Médico Otorrinolaringologista do Hospital Santa Casa de Misericórdia de Ponta Grossa.

6. Médico Radiologista do Serviço de Imagem do Hospita Santa Casa de Misericórdia de Ponta Grossa.

7. Cirurgiã-dentista graduada pela Universidade Estadual de Ponta Grossa.

8. Acadêmico do $4^{\circ}$ período do Curso de graduação em Odontologia das Faculdades Integradas do Centro de Ensino Superior dos Campos Gerais.

9. Coordenador do Curso de Pós-graduação em Ciências da Saúde do Hospital Heliópolis.

Endereço para correspondência: Dr. Ademar José de Oliveira Paes Junior. Rua Cônego Xavier, 276, 10ํandar, Bairro Sacomã. São Paulo, SP, 04231-030. E-mail: ademarnet@yahoo.com

Recebido para publicação em 22/10/2003. Aceito, após revisão, em 12/11/2003.
}

\section{INTRODUÇÃO}

A anomalia do complexo estilo-hióideo, historicamente, passa por três períodos distintos ${ }^{(\mathbf{1})}$. O primeiro período teve início há cerca de 300 anos, quando Pietro Marchetti, um cirurgião de Pádua, em 1652, descreveu o primeiro relato de caso de ossificação do ligamento estilo-hióideo, e passa pela descrição de Eagle, que definiu a estilalgia como entidade autônoma relacionada a uma anormalidade do tamanho do processo estilóide ou à mineralização do complexo do ligamento estilo-hióideo. O segundo período corresponde ao desenvolvimento do diagnóstico radiográfico, quando Grossman correlacionou o complexo de dores, incluindo disfagia, otalgia, estilalgia, cefaléia, dor na articulação têmporo-mandibular e várias formas de dor facial com o alongamento do processo estilóide. O terceiro período corresponde ao advento da radiografia panorâmica e da tomografia computadorizada, as quais permitem a visualização de diversas estruturas do complexo maxilofacial.

O processo estilóide do osso temporal corresponde a uma delgada projeção óssea de aproximadamente $25 \mathrm{~mm}$ de comprimento. Tem sua localização entre as artérias carótidas interna e externa, posteriormente à faringe, onde se inserem os músculos estilo-hióideo, estiloglosso e estilofaríngeo, os quais são inervados, respectivamente, pelos nervos facial, hipoglosso e glossofaríngeo ${ }^{(2,3)}$.

$\mathrm{O}$ conjunto formado pelo processo estilóide, ligamento estilo-hióideo e corno menor do osso hióide corresponde ao complexo ou aparelho estilo-hióideo, os quais têm sua origem embriológica na cartilagem de Reichert do segundo arco branquial ${ }^{(4)}$.

As causas do alongamento do processo estilóide não são bem definidas e várias teorias têm sido propostas, como o alongamento congênito decorrente da persistência de um folheto embrionário cartilaginoso, a calcificação do ligamento estilohióideo, resultando na aparência de um processo estilóide alongado, e a formação de tecido ósseo na inserção do ligamento estilo-hióideo.

O diagnóstico diferencial da síndrome de Eagle inclui nevralgias glossofaríngea e trigeminal, arterite temporal, enxaqueca, cefaléia histamínica, síndrome da disfunção- 
dor miofacial, dor secundária a terceiros molares não irrompidos ou impactados, artrite cervical, tumores e próteses dentais ausentes $^{(2,5,6)}$

\section{RELATO DOS CASOS}

Caso 1 - Paciente do sexo feminino, 47 anos de idade, desdentada total superior e inferior, assintomática (Figuras 1 e 2).

Caso 2 - Paciente do sexo feminino, 34 anos de idade, apresentando quadro doloroso grave, já tendo sido submetida a vários tratamentos com neurologistas, médicos generalistas e ortodontistas, durante cinco anos (Figura 3).

\section{DISCUSSÃO}

A síndrome de Eagle corresponde a um agregado de sintomas, incluindo dor facial recorrente, sensação de corpo estranho e disfagia, estando diretamente relacionada ao alongamento do processo estilóide ou à calcificação do ligamento estilo-hióideo. O processo estilóide tem seu tamanho normal muito variável, com relatos de séries de estudos em cadáveres, cujos comprimentos variaram entre $1,52 \mathrm{~cm}$ e $4,77 \mathrm{~cm}$. Nos estudos radiográficos, o processo estilóide é definido como normal quando menor do que $2,5 \mathrm{~cm}$ e estando alongado quando maior do que $4,0 \mathrm{~cm}$, quando a ocorrência da síndrome de Eagle é bastante provável. A variação entre estas medidas é considerada inespecífica, tornando necessário o prosseguimento da investigação por outros meios diagnósticos ${ }^{(\mathbf{7 , 8})}$.

A prevalência de um processo estilóide alongado tem grande variabilidade na população. Eagle, em sua publicação original, relatou o processo estilóide alongado em $4 \%$ dos seus casos, sendo que apenas $4 \%$ destes apresentavam sintomas positivos ${ }^{(\mathbf{6})}$. Esta baixa correlação entre o achado radiográfico do processo estilóide alongado e sintomas específicos foi confirmada nas séries de Kaufman, em 1970, que relatou o alongamento do processo estilóide em

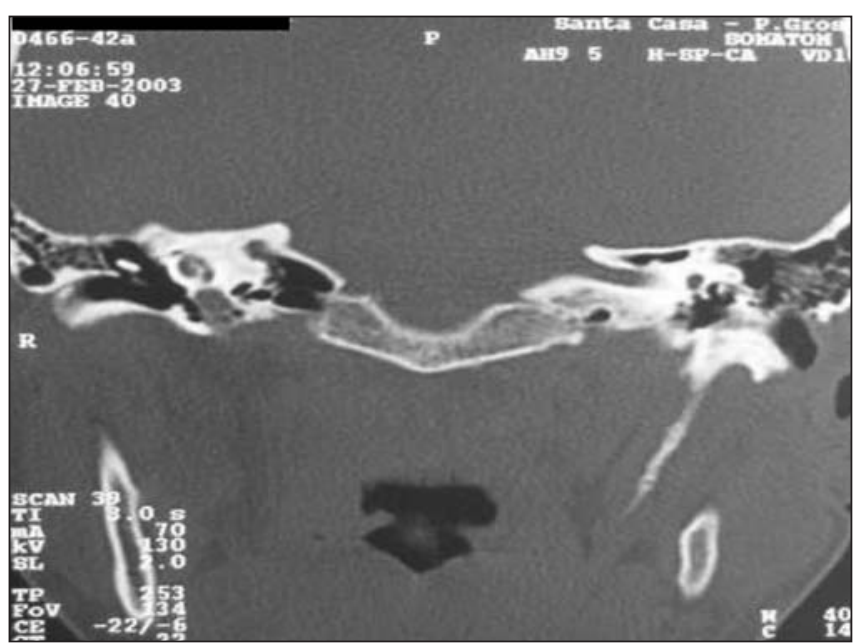

Figura 1. Caso 1. Tomografia computadorizada, corte coronal com janela óssea, mostrando o processo estilóide esquerdo alongado.

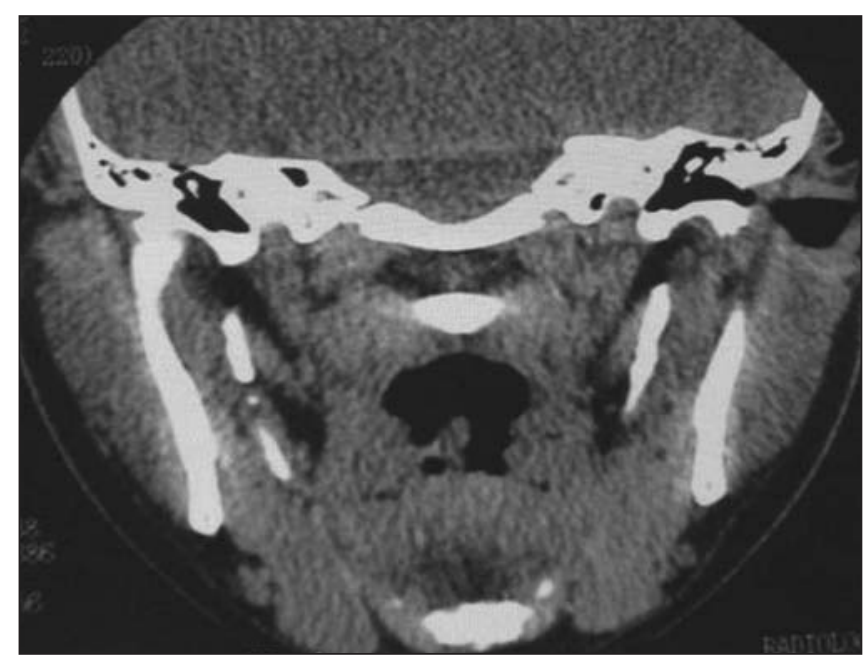

A

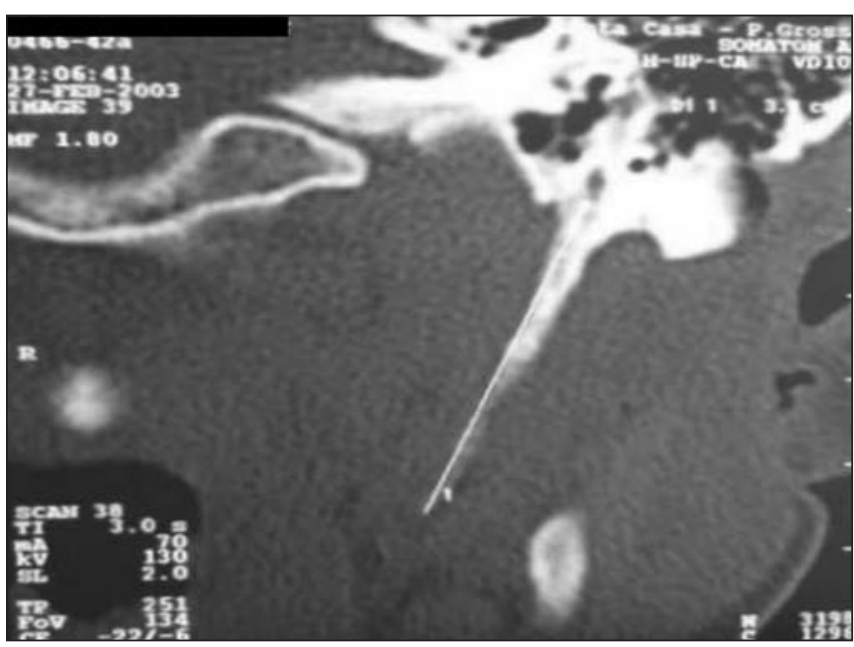

Figura 2. Caso 1. Tomografia computadorizada, corte coronal com janela óssea, com ampliação do processo estilóide esquerdo alongado.

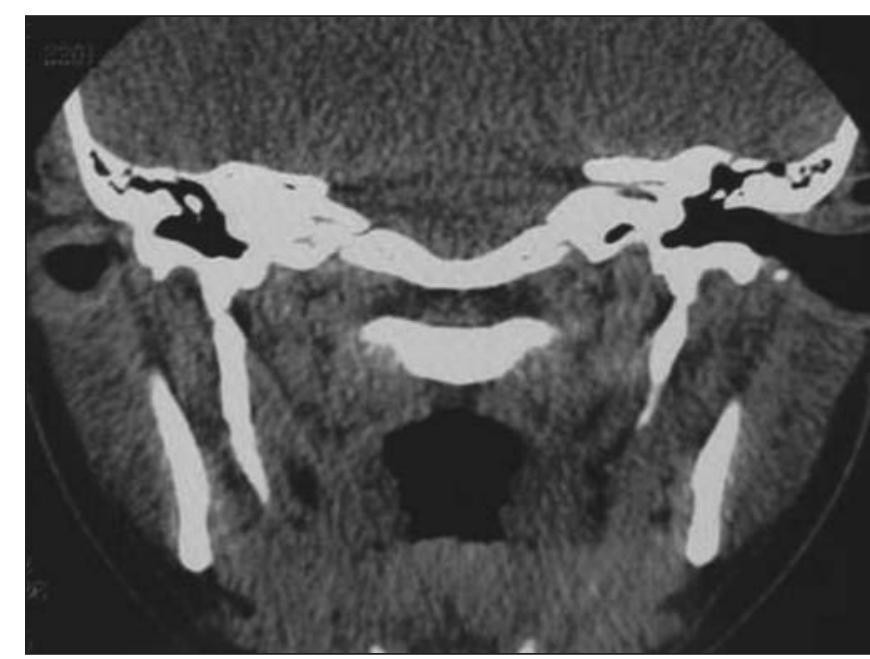

B

Figura 3. Caso 2. Tomografia computadorizada, cortes coronais com janela para tecidos moles, demonstrando os processos estilóides, à direita estando alongado e espessado e à esquerda tendo aspecto normal. Notar a boa resolução das relações dos processos estilóides com as estruturas adjacentes, algo que a tomografia computadorizada permite avaliar. 
7,3\% dos seus pacientes ${ }^{(\mathbf{9})}$. Correl, em 1979 examinou 1.700 radiografias panorâmicas e observou alongamento do processo estilóide ou calcificação do complexo estilohióideo em $18 \%$ dos casos, verificando baixa concordância com a presença de sintomas. Isto nos leva a concluir que a maioria dos pacientes com processo estilóide alongado é assintomática ${ }^{(2,9)}$.

Inversamente, muitos pacientes com sinais e sintomas sugestivos da síndrome de Eagle não apresentam evidências radiográficas de um processo alongado, fato verificado por Steimann, que relatou 30 pacientes com quadro clínico positivo, dos quais 26 não apresentavam alongamento do processo estilóide ou calcificação do ligamento estilo-hióideo ${ }^{(9)}$.

Diante do exposto, os indivíduos afetados por processo estilóide alongado raramente desenvolvem sintomas, entretanto, quando presentes, podem incluir dor em qualquer dos lados da garganta, com ou sem irradiação ao ouvido ou região mastóidea do lado afetado. Disfagia, sensação de corpo estranho na garganta, glossalgia, disfonia, cefaléia recorrente, carotidínia, vertigem, perturbações visuais e restrições no movimento do pescoço podem completar o quadro clínico ${ }^{(6,9)}$.

O diagnóstico desta entidade pode ser confirmado clinicamente com a palpação cuidadosa da fossa tonsilar, com relato de dor pelo paciente, associado ao encontro dos sinais radiológicos sugestivos ${ }^{(\mathbf{9})}$.

$\mathrm{O}$ aperfeiçoamento dos meios diagnósticos observados no século XX teve importante participação no estabelecimento dos sinais radiológicos característicos da síndrome de Eagle.

O processo estilóide alongado pode ser visto nos métodos de imagem em incidências radiográficas da face em perfil, ântero-posteriores e oblíquas, porém a tomografia computadorizada é considerada o método de escolha para esta avaliação. A radiografia panorâmica também fornece importantes informações. A tomografia computadorizada tem papel fundamental na avaliação do complexo estilo-hióideo, por permitir a aquisição de imagens nos planos axial e coronal, a realização de reconstruções multiplanares e tridimensionais, demonstrando não somente as estruturas ósseas e os componentes calcificados, como nos estudos radiográficos, mas também a relação destas com as demais estruturas anatômicas adjacentes, superando algumas limitações da radiologia convencional ${ }^{(\mathbf{7 , 8})}$.

\section{CONCLUSÃO}

O diagnóstico da síndrome de Eagle é estabelecido pela associação de dados de história clínica, exame físico e exames complementares.

Exercem papel fundamental na avaliação destes pacientes a radiografia panorâmica e, principalmente, a tomografia computadorizada. Esta última permite a avaliação conjunta do complexo estilo-hióideo e das estruturas anatômicas adjacentes.

\section{REFERÊNCIAS}

1. Gossman JR Jr, Tarsitano JJ. The styloid-stylohyoid syndrome. J Oral Surg 1977;35:555-60.

2. Glogoff MR, Baum SM, Cheifetz I. Diagnosis and treatment of Eagle's syndrome. J Oral Surg 1981; 39:941-4.

3. Baddour HM, McAnear JT, Tilson HB. Eagle's syndrome. Report of a case. Oral Surg Oral Med Oral Pathol 1978;46:486-94.

4. Balbuena L Jr, Hayes D, Ramirez SG, Johnson R. Eagle's syndrome (elongated styloid process). South Med J 1997;90:331-4.

5. Godden DR, Adam S, Woodwards RT. Eagle's syndrome: an unusual cause of a clicking jaw. Br Dent J 1999;186:489-90.

6. Quereshy FA, Gold ES, Arnold J, Powers MP Eagle's syndrome in an 11-year-old patient. J Oral Maxillofac Surg 2001;59:94-7.

7. Murtagh RD, Caracciolo JT, Fernandez G. CT findings associated with Eagle syndrome. AJNR 2001; 22:1401-2.

8. Fini G, Gasparini G, Filippini F, Becelli R, Marcotullio D. The long styloid process syndrome or Eagle's syndrome. J Craniomaxillofac Surg 2000; 28:123-7.

9. Moraes S, Nakonechnyj P, Chaia A. Síndrome de Eagle. Relato de um caso. Rev Bras Odont 1991; XLVIII:30-5. 